\title{
Frailty, multimorbidity and in-hospital cardiopulmonary resuscitation: predictable markers of outcome?
}

\author{
Authors: Elin H Thomas, ${ }^{A}$ Aled R Lloyd ${ }^{B}$ and Nicky Leopold ${ }^{C}$
}

\section{Background}

This study's aim was to investigate an association between outcome from in-hospital cardiopulmonary resuscitation (CPR) and increasing burden of comorbidities and frailty.

\section{Methods}

Retrospective analysis of prospectively collected data from contemporaneous patient notes and electronic records of all patients who suffered an in-hospital cardiac arrest between 1 April 2017 and 31 March 2018 in a hospital that includes a tertiary cardiology department.

Results

A total of 113 patient records were assessed. Patient frailty was assessed based on calculation of Rockwood clinical frailty score (CFS) and comorbidity assessment based on Charlson comorbidity index (CCI). A linear correlation has been identified between increasing $\mathrm{CCI}$ and reduced survival (ANOVA $=p<0.001)$ and rates of return of spontaneous circulation (ROSC) (ANOVA $=0.013$ ). No patients with a CFS above 6 survived to 1 year. A linear correlation was identified between increasing CFS and reduced probability of ROSC (ANOVA $p=0.002$ ), survival to discharge (ANOVA $p=0.003$ ) and 1 year (ANOVA $p=0.001$ ).

Conclusion

Our findings suggest an association between increasing patient multimorbidity and frailty and poorer outcome post cardiac arrest.

KEYWORDS: frailty, cardiopulmonary resuscitation, multimorbidity, Rockwood, Charlson

DOI: $10.7861 /$ clinmed.2020-1002

\section{Introduction}

Despite the obvious potential benefits, cardiopulmonary resuscitation (CPR) is an invasive and often harmful treatment intervention. ${ }^{1}$ As a result of COVID-19, it is also increasingly

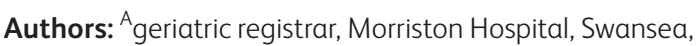
UK; ${ }^{B}$ nephrology registrar, Morriston Hospital, Swansea, UK; ${ }^{\mathrm{C}}$ consultant geriatrician, Singleton Hospital, Swansea Bay University Health board, Swansea, UK
}

recognised as a danger to the personnel who perform CPR. ${ }^{2}$ Incidences of adult in-hospital cardiac arrests have been demonstrated to occur in 1.6/1,000 hospital admissions and survival to hospital discharge shown in a large prospective analysis of UK National Cardiac Arrest Audit (NCAA) database to be as low as $18.4 \%{ }^{3}$ Factors such as age ${ }^{4}$ and presenting cardiac rhythm ${ }^{1,5}$ have been identified as factors that influence the likelihood of survival post-CPR, but little is known regarding the impact of the patient's frailty on their outcome post-CPR.

Frailty has been described as 'a clinical state characterised by a decrease of an individual's homeostatic reserves and is responsible for enhanced vulnerability to endogenous and/or exogenous stressors'. 6 It has been quantified by Rockwood et al, and their nine-point scale has been extensively used in research to objectively assess the impact increasing frailty has on medical interventions. ${ }^{7}$ Many studies have demonstrated an association between increasing frailty and a worse outcome in a variety of different fields ${ }^{8,9}$ but our knowledge of the impact of frailty on CPR outcomes remains sparse. Similarly, increasing disease burden has been shown to influence outcome in a variety of surgical and medical fields, ${ }^{10,11}$ and the Charlson comorbidity index ${ }^{12}$ (CCI) has proved a useful tool to measure age-adjusted disease burden and as a prognostic indicator for mortality. As with frailty, the sparse research undertaken already has also demonstrated a link between worse outcome from CPR and increasing comorbidity. ${ }^{13-15}$ It is not clear if there is a linear correlation between incremental increase in frailty and multimorbidity and reduced likelihood of achieving return of spontaneous circulation (ROSC) or improving mortality. The impact of a primary percutaneous coronary intervention (PCI) centre on subsequent results has also not been well established. The aim of this project is to answer these questions and to add to the growing body of evidence in this field.

\section{Methods}

A retrospective analysis of prospectively collected data from contemporaneous patient notes and electronic patient records was undertaken. A comprehensive list of all in-hospital cardiac arrests between 1 April 2017 and 31 March 2018 in a tertiary hospital in South Wales was obtained from the in-hospital resuscitation team that routinely and prospectively collects and stores this data. The study population included all adults (aged over 16) who suffered an in-hospital cardiac arrest during the study period. If a patient suffered recurrent cardiac arrests during an admission, only the first arrest was included and 
Fig 1. Rookwood Clinical Frailty Scale. ${ }^{7}$ IADL $=$ instrumental activities of daily living. Reproduced with permission from Geriatric Medicine Research, Dalhousie University.

\section{Clinical Frailty Scale*}

I Very Fit - People who are robust, active, energetic and motivated. These people commonly exercise regularly. They are among the fittest for their age.

i, 2 Well-People who have no active disease symptoms but are less fit than category I. Often, they exercise or are very active occasionally, e.g. seasonally.

3 Managing Well - People whose medical problems are well controlled, but are not regularly active

beyond routine walking.

1

4 Vulnerable-While not dependent on others for daily help, often symptoms limit activities. A common complaint is being "slowed up", and/or being tired during the day.

5 Mildly Frail - These people often have more evident slowing, and need help in high order IADLs (finances, transportation, heavy housework, medications). Typically, mild frailty progressively impairs shopping and walking outside alone, meal preparation and housework

6 Moderately Frail - People need help with all outside activities and with keeping house. Inside, they often have problems with stairs and need help with bathing and might need minimal assistance (cuing, standby) with dressing
I 7 Severely Frail-Completely dependent for personal care, from whatever cause (physical or cognitive). Even so, they seem stable and not at high risk of dying (within $\sim 6$ months).

8 Very Severely Frail - Completely dependent approaching the end of life. Typically, they could not recover even from a minor illness.

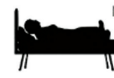

9.Terminally III - Approaching the end of life.This category applies to people with a life expectancy $<6$ months, who are not otherwise evidently frail.

\section{Scoring frailty in people with dementia}

The degree of frailty corresponds to the degree of dementia. Common symptoms in mild dementia include forgetting the details of a recent event, though still remembering the event itself, repeating the same question/story and social withdrawal.

In moderate dementia, recent memory is very impaired, even though they seemingly can remember their past life events well. They can do personal care with prompting.

In severe dementia, they cannot do personal care without help.

* I. Canadian Study on Health \& Aging, Revised 2008. 2.K. Rockwood et al. A global clinical measure of fitness and frailty in elderly people. CMA] 2005; 173:489-495. Q 2007-2009. Version 1.2All ights reserved. Geriatric Mecicine Research, Dahouse University, Halifix Canada
to copy for research and educational purposes recorded in the analysis. Patients who suffered an out-of-hospital cardiac arrest (OOHCA) and those found during the resuscitation efforts to have a do not attempt cardiopulmonary resuscitation (DNACPR) order were excluded. Case records for each patient were reviewed to ascertain basic demographic details and information regarding the admitting specialty, arrest rhythm, duration of CPR and achievement of ROSC. Electronic patient records were analysed to assess 30-day survival, 1-year survival and survival to discharge. No information was gathered regarding the need for an ITU admission following ROSC.

Patient frailty was calculated using the Rockwood clinical frailty scale (CFS) (Fig 1) based on the documented clerking social and functional histories. Notes were independently assessed by two members of the research team and where there was doubt between two frailty scale points, the lowest was allocated. If no social history was recorded or the data were inadequate to determine a Rockwood CFS, they were excluded from the frailty analysis. Multimorbidity was similarly assessed using patient records and subsequent calculation of the CCI. Those with no recorded list of comorbidities were also excluded from the relevant analyses. Statistical analysis and non-parametric tests were performed using SPSS Statistics (Version 27). As per direction from the hospital research and development department, ethical approval was not required for this project.

\section{Results}

Patient characteristics and study results are summarised in Tables 1 and 2 .

A total of 192 cardiac arrest calls were made between 1 April 2017 and 31 March 2018. After the exclusion criteria were applied (Fig 2), 113 patients were included in the data analysis. Twentysix patients were excluded as it was not possible to locate their contemporaneous clinical notes, while another 28 were excluded as they did not appear to have received CPR. Adequate comorbidity data were extracted from the notes of 108 patients to allow

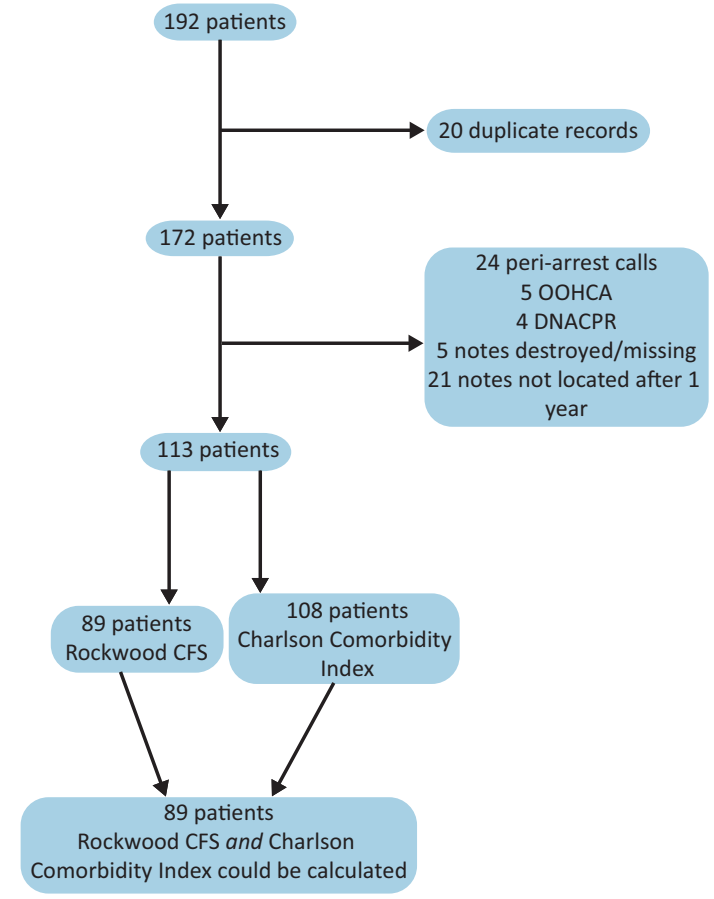

Fig 2. Flow chart of included patients. CFS = clinical frailty scale; DNACPR = do not attempt cardiopulmonary resuscitation; OOHCA = out-of-hospital cardiac arrest.

analysis of CPR outcomes against CCI. Sufficient data regarding the functional status of the patients allowing a Rockwood frailty scale to be calculated were possible for 89 patients.

The average age of these patients was 73.6 , with a range from 21 to 97 years old. $69.9 \%$ of the patients were male. The overall 


\begin{tabular}{|c|c|c|c|c|}
\hline & $\begin{array}{l}\text { Total number } \\
\text { of patients }\end{array}$ & CFS & $\begin{array}{l}\text { Charlson } \\
\text { CI }\end{array}$ & $\begin{array}{l}\text { CFS and } \\
\text { Charlson CI }\end{array}$ \\
\hline \multicolumn{5}{|l|}{ Age } \\
\hline$<40$ & 1 & 1 & 1 & 1 \\
\hline $40-49$ & 3 & 2 & 2 & 2 \\
\hline 50-59 & 9 & 8 & 9 & 8 \\
\hline $60-69$ & 19 & 16 & 19 & 16 \\
\hline 70-79 & 47 & 36 & 45 & 36 \\
\hline 80-89 & 30 & 23 & 29 & 23 \\
\hline 90-99 & 4 & 3 & 4 & 3 \\
\hline \multicolumn{5}{|l|}{ Specialty } \\
\hline $\begin{array}{l}\text { General } \\
\text { medicine }\end{array}$ & 41 & 40 & 44 & 40 \\
\hline Cardiology & 44 & 30 & 40 & 30 \\
\hline Nephrology & 3 & 3 & 3 & 3 \\
\hline $\begin{array}{l}\text { General } \\
\text { surgery }\end{array}$ & 14 & 7 & 14 & 7 \\
\hline $\begin{array}{l}\text { ENT, T\&O, } \\
\text { vascular }\end{array}$ & 11 & 10 & 11 & 10 \\
\hline
\end{tabular}

CFS = clinical frailty scale; CI = comorbidity index; ENT = ear, nose and throat; T\&O = trauma and orthopaedic surgery.

rate of ROSC in the 113 patients was $62.0 \%$, while the overall survival at discharge and 30 days was $25 \%$ and the overall 1 -year survival was $24.1 \%$.

Out of the total cardiac arrest events studied, $24.8 \%$ of cases presented in a shockable ECG rhythm. Sixty-three percent of the patients presenting in a shockable rhythm survived to discharge compared with only $12 \%$ who presented in a non-shockable rhythm.

\section{Rockwood CFS assessments}

ROSC

When data collected for the 89 patients with adequate information to calculate a Rockwood CFS were analysed, a linear correlation was identified between increasing CFS and reduced rates of ROSC (ANOVA $p=0.002$ ). When the rates of ROSC of the frail population (generally accepted in the literature ${ }^{7}$ as a Rockwood CFS of 5 and above) were compared to the rest of the study population, they were found to be lower (chi-squared $p<0.001)$. This remained true when vulnerable / pre-frail patients (CFS 4) were included in the frail population or if a Rockwood CFS of 6 was used as the definition of frailty (chi-squared $p<0.001$ for both).

\section{Survival}

Of the patients who survived to either 30 days or to discharge, their likelihood of surviving to 1 year post cardiac arrest was good. Only one patient survived to discharge but not to 1 year.
A linear trend was identified between increasing Rockwood CFS and reduced survival to discharge (ANOVA $p=0.003$ ), 30 days (ANOVA $p=0.003$ ) and 1 year (ANOVA $p=0.001$ ).

When the frail population in this study was compared to the remainder of the study population, there was a significant difference in all forms of survival. As with the ROSC data, this remained the same if a Rockwood CFS of 4, 5 or 6 was used as the definition of frailty (chi-squared $p<0.001$ for all three).

No patients with a Rockwood CFS above 6 in this study survived to 1 year after cardiac arrest.

\section{CCI assessments}

\section{ROSC}

On analysing the comorbidity data collected from 108 patients, a linear correlation was identified between an increasing CCI and a reduced probability of ROSC (ANOVA $p=0.013$ ).

\section{Survival}

A linear relationship was also identified between increasing CCI and survival to discharge, 30 days and 1 year (ANOVA $p<0.001$ for all three measures).

No patient in this study with a CCI of greater than 6 survived for 1 year after their cardiac arrest.

\section{Cardiology patients}

A subgroup analysis was performed of patients under the care of the cardiology department. In this population, $52.4 \%$ of the cardiac arrest events presented with a shockable ECG rhythm. No data were collected on the proportion of these cardiac events that occurred during a procedure or their location within the cardiology department.

Excluding cardiology patients, the overall rate of survival to discharge, 30 days and 1 year in this study was $10.2 \%$ for all three parameters with $55.9 \%$ of patients returning to spontaneous circulation after CPR. For cardiology patients, the overall rates of survival to discharge, 30 days and 1 year in this study were $46.6 \%$, $46.6 \%$ and $44.4 \%$, respectively, with $76.7 \%$ of patients returning to spontaneous circulation after CPR.

\section{Rockwood CFS}

Thirty patients under the care of cardiology were included in the Rockwood CFS analysis. This patient group had an average survival to discharge rate of $56.7 \%$ and a rate of ROSC of $86.7 \%$, significantly better than the overall study population. A high rate of ROSC was observed in these patients across all groups of frailty, but survival rates generally dropped as frailty increased. No significant relationship was identified between increasing Rockwood CFS and ROSC ( $p=0.573), 30$-day survival $(p=0.356)$, survival to discharge $(p=0.356)$ or 1 year $(p=0.060)$.

\section{CCI}

Forty patients under the care of cardiology were included in the CCI analysis. Overall ROSC was observed in $80 \%$ of these patients and the average survival rate to discharge was $50 \%$. In a similar trend seen to the Rockwood CFS analysis, rates of ROSC remained high across all groups but survival decreased with increasing CCI. For these patients, no relationship was identified between ROSC and increasing CCI $(p=0.273)$; however, a statistically significant 
Table 2. Summary of results

Total

\section{Clinical frailty scale pooled}

1-3 (not frail)

4 (pre-frail)

5-9 (frail)

\section{Cardiology patients clinical frailty scale pooled}

1-3 (not frail)

4 (pre-frail)

5-9 (frail)

Total

ANOVA p value

Non-cardiology patients clinical frailty scale pooled

1-3 (not frail)

4 (pre-frail)

5-9 (frail)

Total

\section{Charlson (pooled)}

0

$1-3$

$>4$

\section{Charlson (pooled) cardiology patients}

0

$1-3$

$>4$

Charlson (pooled) non-cardiology patients

0

$1-3$

$>4$

ROSC $=$ return of spontaneous circulation

\begin{tabular}{|c|c|c|c|c|}
\hline $\begin{array}{l}\text { Number of } \\
\text { patients }\end{array}$ & ROSC & $\begin{array}{l}\text { 30-day } \\
\text { survival }\end{array}$ & $\begin{array}{l}\text { Survival to } \\
\text { discharge }\end{array}$ & $\begin{array}{l}\text { 1-year } \\
\text { survival }\end{array}$ \\
\hline 113 & $68(60.1 \%)$ & $27(23.9 \%)$ & $27(23.9 \%)$ & $26(23.0 \%)$ \\
\hline 35 & $30(85.7 \%)$ & $16(45.7 \%)$ & $16(45.7 \%)$ & $16(45.7 \%)$ \\
\hline 15 & $8(53.3 \%)$ & $3(20.0 \%)$ & $3(20.0 \%)$ & $3(20.0 \%)$ \\
\hline 39 & $21(58 \%)$ & $5(12.8 \%)$ & $5(12.8 \%)$ & $4(10.3 \%)$ \\
\hline 18 & 17 & $13(72.2 \%)$ & $13(72.2 \%)$ & $13(72.2 \%)$ \\
\hline 6 & 3 & $2(33.3 \%)$ & $2(33.3 \%)$ & $2(33.3 \%)$ \\
\hline 6 & 6 & $3(50.0 \%)$ & $3(50.0 \%)$ & $2(33.3 \%)$ \\
\hline \multirow[t]{2}{*}{30} & $26(86.7)$ & $18(60.0 \%)$ & $18(60.0 \%)$ & $17(56.7 \%)$ \\
\hline & 0.573 & 0.356 & 0.356 & 0.060 \\
\hline 17 & $13(76.5 \%)$ & $3(17.6 \%)$ & $3(17.6 \%)$ & $3(17.6 \%)$ \\
\hline 9 & $5(55.6 \%)$ & $1(11.1 \%)$ & $1(11.1 \%)$ & $1(11.1 \%)$ \\
\hline 33 & $15(45.5 \%)$ & $2(6.1 \%)$ & $2(6.1 \%)$ & $2(6.1 \%)$ \\
\hline 59 & $33(55.9 \%)$ & $6(10.2 \%)$ & $6(10.2 \%)$ & $6(10.2 \%)$ \\
\hline 1 & $1(100 \%)$ & $1(100 \%)$ & $1(100 \%)$ & $1(100 \%)$ \\
\hline 18 & $14(77.8 \%)$ & $8(44.4 \%)$ & $8(44.4 \%)$ & $8(44.4 \%)$ \\
\hline 89 & $52(58.4)$ & $18(20.2 \%)$ & $18(20.2 \%)$ & $17(19.1 \%)$ \\
\hline 1 & $1(100 \%)$ & $1(100 \%)$ & $1(100 \%)$ & $1(100 \%)$ \\
\hline 7 & $7(100 \%)$ & $7(100 \%)$ & $7(100 \%)$ & $7(100 \%)$ \\
\hline 31 & $24(77.4 \%)$ & $14(45.2 \%)$ & $14(45.2 \%)$ & $13(41.9 \%)$ \\
\hline 0 & 0 & 0 & 0 & 0 \\
\hline 11 & $7(63.6 \%)$ & $1(9.1 \%)$ & $1(9.1 \%)$ & $1(9.1 \%)$ \\
\hline 58 & $10(17.2 \%)$ & $4(6.9 \%)$ & $4(6.9 \%)$ & $4(6.9 \%)$ \\
\hline
\end{tabular}

trend was identified between increasing CCI and mortality and 30 days $(p=0.002)$, discharge $(p=0.002)$ and 1 year $(p<0.001)$.

\section{Age}

Increasing age was linearly associated with both an increasing Rockwood frailty scale (ANOVA $p<0.001$ ) and an increased CCI (ANOVA $p<0.001$ ). Increasing age was found to be linearly associated with increased mortality at discharge, 30 days and 1 year (ANOVA $p<0.001$ for all three) and with reduced likelihood of ROSC.

\section{Duration of CPR}

No linear relationship was identified between age and duration of CPR (ANOVA $p=0.376)$, CCI and duration of CPR (ANOVA $p=0.112)$.

\section{Discussion}

Our research adds to the growing body of evidence that links worsening frailty with increasingly adverse outcome from inhospital CPR. Worldwide, Smith et al's Australian retrospective 10-year analysis first demonstrated a decreased likelihood of discharge from hospital for the frailest patients, but could not demonstrate an independent association with worsened survival. ${ }^{16}$ This publication used the Hospital Frailty Risk Score (HFRS) to calculate patient frailty. The retrospective reviews of Ibitoye et $a l^{14}$ and Wharton et $a l^{13}$ are two other publications assessing the relationship between frailty and CPR outcome that have been published since we began collecting our data. Both have demonstrated a reduced likelihood of survival to discharge with a Rockwood CFS of above 5 and 6 , respectively. They both also used the Rockwood frailty scale as their measurement of frailty, thus 
direct comparisons with our data could more easily be made. Our population's overall survival to discharge also aligned with these publications at $25 \%$.

Like our research, Wharton et al, ${ }^{13}$ for example, also did not exclude participants on the basis of age and similarly demonstrated that the likelihood of achieving ROSC and survival to hospital discharge worsened with a higher CFS. The findings of Ibitoye et al's retrospective review of inpatient CPR $^{14}$ also correlated broadly with our own findings of worsening survival to discharge but their review was limited to those aged 60 or above. As both authors grouped their results for analysis, we cannot determine the impact each incremental increase in CFS had on in-hospital CPR outcome.

Unlike other research in this field, we analysed the effect of each incremental increase in CFS on survival to discharge, 30-day and 1-year survival. Significantly, our research suggests that there exists a linear correlation between worsening CPR survival (to ROSC, discharge, 30 days and 1 year) and increasing frailty. Such a linear trend was statistically significant and emphasises the vital importance of ascertaining an accurate assessment of pre-admission function and ability (physically, cognitively and socially) to correctly assign a patient's CFS. Armed with accurate information and knowledge about the incremental worsening of outcome associated with CPR, better informed best interest decisions and patient discussions can thus be had. There also seems to be a significant difference in outcome associated with a CFS greater than 6 . For example, no patients in our study with a CFS of equal to or greater than 6 survived 1 year but $22 \%$ of those with a CFS of 5 and $20 \%$ of those with a CFS of 4 survived to 1 year.

Significantly, our assessment of the association between increasing disease burden and CPR survival also showed a statistically significant linear correlation between increased disease burden and poor CPR outcome. Reduced survival and rates of ROSC were noted for individuals with a CCI above 3 . This is especially true for those with a CCI greater than 6, as none of this group in our analysis survived to 1 year post cardiac arrest. Accurate establishment of past medical history thus also seems imperative when deciding on the appropriateness of CPR too.

Uniquely compared to other recently published research in this field that followed outcome only to hospital discharge, we followed our population for 1 year following their cardiac arrest to better understand the longer-term outcomes. Interestingly, this research suggests that those who survive to discharge are very likely to survive to 1 year. All but one of those who survived to discharge survived to 1 year.

We also aimed to assess the impact of a PCI centre on the results. Our results suggest that the overall survival to ROSC/ discharge/30 days and 1-year survival was better in those admitted under the primary care of the cardiologists compared to all other specialties. We hypothesise that this difference may reflect the higher number of patients admitted under this specialty with shockable rhythms $(69.7 \%$ vs $25 \%$ in the rest of the population) for which survival is more likely. ${ }^{17}$ These patients were also more likely to arrest in a favourable environment for resuscitation eg, catheterisation lab/CCU. We could not determine a linear trend between increased frailty and poorer outcomes in this population. This could be as a result of a higher proportion of shockable arrest rhythms in this population with associated improved outcomes, even for frailer patients. However, only 30 patients were included in this group and only six were frail and six were pre-frail; therefore, further research is required with larger sample sizes to ascertain more definitive associations. We feel that these results are representative of those that would be expected in other centres with primary PCI on site. We believe that the results excluding cardiology patients are reasonably representative of those that could be expected for patients who do not require cardiac intervention in other settings.

We feel that our research adds to a growing body of evidence in this field and adds new insights. However, we recognise the limitations of a single-site retrospective study and the limitations that result from the incomplete dataset caused by missing notes and an absence of adequate functional or social history taken by admitting teams. Nevertheless, these limitations themselves offer valuable learning opportunities and observations. That we could not obtain the relevant notes of $15 \%$ of our targeted population, for example, raises questions about the reliability of the way our patient records are kept. Our results also suggest that doctors clerking from a medical team are proportionately much more likely to take a functional and social history of sufficient detail to reliably calculate a CFS. Such information being readily available in the patients' notes is of paramount importance for pressurised decision making in emergency situations such as cardiac arrests when decisions have often had to be made acutely based only on the information provided by the admitting team. This, of course, has become of particular relevance during the COVID-19 pandemic.

We also observed that the decision to resuscitate was seemingly made by omission in many cases whereby there was no documented evidence that a do not attempt cardiopulmonary resuscitation (DNACPR) had even been considered or discussed. Our research population, for example, included patients with known advanced metastatic solid organ cancers, those with a left ventricular ejection fraction calculated at less than $10 \%$, patients who on inspection of earlier admission notes had refused CPR and even those for whom a DNACPR order simply didn't follow them from intensive care to their step-downward. Such occurrences emphasise the importance of early discussions with patients/families/ colleagues about the appropriateness of medical interventions. Following a national culture shift towards early decision making during the COVID pandemic, perhaps such scenarios are confined to a pre-COVID era.

Despite these insights and the knowledge, we add to this field, a large prospective multicentre trial would of course be very beneficial in proving a definitive association between increasing frailty and poorer survival post-cardiac arrest.

\section{Conclusion}

Our findings add to a growing body of evidence demonstrating an association between increasing patient frailty and poorer outcome post in-hospital cardiac arrest. Our research suggests that there is a linear correlation between both increasing clinical frailty and multimorbidity with poorer CPR outcome. In this study, those who did survive to discharge were also very likely to survive to at least 1 year. This is a single-centre retrospective analysis and more research is required in this field. 
Summary

\section{What is known?}

Increased frailty adversely affects outcome in a wide range of medical interventions including dialysis and surgical procedures. Little is known about the association between frailty, multimorbidity and outcomes from in-hospital CPR. Of the data already available, only grouped increases in clinical frailty scale (CFS) scores have been analysed. Similarly, there is little evidence regarding worsening outcome from CPR with increasing multimorbidity. The impact of having an on-site primary percutaneous intervention centre on CPR outcomes in frailer patients is also relatively unknown.

\section{What is the question?}

The study aimed to assess whether there is a linear relationship between increases in patients' CFS or CCI (a score of multimorbidity) and resultant outcome from in-hospital CPR. We also followed all patients for 1 year post cardiac arrest to assess longer-term outcome. We also assessed whether the presence of an on-site primary percutaneous treatment centre influenced outcome.

\section{What was found?}

A linear correlation was identified between increasing CCI with both reduced survival and rates of ROSC. A linear correlation was also identified between increasing CFS and reduced probability of ROSC, survival to discharge and to 1 year.

\section{What is the implication for practise now?}

These results add to the growing evidence in the field that frailty and multimorbidity adversely affect outcome from CPR. Comprehensive and accurate social and functional histories should be considered as even more important factors and trigger early discussions regarding the appropriateness of CPR as each incremental increase in CFS could tip the risk/benefit balance.

\section{Supplementary material}

Additional supplementary material may be found in the online version of this article at www.rcpjournals.org/clinmedicine: S1 - Full summary of results.

\section{References}

1 Deliliga A, Chatzinikolaou F, Koutsoukis D, Chrysovergis I, Voultsos P. Cardiopulmonary resuscitation (CPR) complications encountered in forensic autopsy cases. BMC Emerg Med 2019;19:23.

2 Mahase E, Kmietowicz Z. Covid-19: Doctors are told not to perform CPR on patients in cardiac arrest. BMJ 2020;368:m1282.
3 Nolan JP, Soar J, Smith GB et al. Incidence and outcome of in-hospital cardiac arrest in the United Kingdom National Cardiac Arrest Audit. Resuscitation 2014;85:987-92.

4 Hirlekar G, Karlsson T, Aune S et al. Survival and neurological outcome in the elderly after in-hospital cardiac arrest. Resuscitation 2017:118:101-6.

5 Woolcott OO, Reinier K, Uy-Evanado A et al. Sudden cardiac arrest with shockable rhythm in patients with heart failure. Heart Rhythm 2020;17:1672-78.

6 Cesari M, Calvani R, Marzetti E. Frailty in older persons. Clin Geriatr Med 2017:33:293-303.

7 Rockwood K, Song X, Macknight C et al. A global clinical measure of fitness and frailty in elderly people. CMAJ 2005;173:489-95.

8 Wallis SJ, Wall J, Biram RWS, Romero-Ortuno R. Association of the clinical frailty scale with hospital outcomes. QJM 2015;108:943-9.

9 Alfaadhel TA, Soroka SD, Kiberd BA, et al. Frailty and mortality in dialysis: evaluation of a clinical frailty scale. Clin J Am Soc Nephrol 2015;10:832-40.

10 Birim O, Kappetein AP, Bogers AJJC. Charlson comorbidity index as a predictor of long-term outcome after surgery for nonsmall cell lung cancer. Eur J Cardiothorac Surg 2005;28:759-62.

11 Ofori-Asenso R, Zomer E, Chin KL et al. Effect of comorbidity assessed by the Charlson comorbidity index on the length of stay, costs and mortality among older adults hospitalised for acute stroke. Int J Environ Res Public Health 2018;15:2532. www.mdpi. com/1660-4601/15/11/2532

12 Charlson ME, Pompei P, Ales KL, MacKenzie CR. A new method of classifying prognostic comorbidity in longitudinal studies: development and validation. J Chronic Dis 1987:40:373-83.

13 Wharton C, King E, MacDuff A. Frailty is associated with adverse outcome from in-hospital cardiopulmonary resuscitation. Resuscitation 2019;143:208-11.

14 Ibitoye SE, Rawlinson S, Cavanagh A, Phillips V, Shipway DJH. Frailty status predicts futility of cardiopulmonary resuscitation in older adults. Age Ageing 2021;50:147-152.

15 Piscator E, Hedberg P, Göransson K, Djärv T. Survival after inhospital cardiac arrest is highly associated with the age-combined Charlson co-morbidity index in a cohort study from a two-site Swedish University hospital. Resuscitation 2016;99:79-83.

16 Smith RJ, Reid DA, Santamaria JD. Frailty is associated with reduced prospect of discharge home after in-hospital cardiac arrest. Internal Med J 2019:49:978-985.

17 Meaney PA, Nadkarni VM, Kern KB et al. Rhythms and outcomes of adult in-hospital cardiac arrest. Crit Care Med 2010;38:101-8. https://journals.lww.com/ccmjournal/Fulltext/2010/01000/ Rhythms_and_outcomes_of_adult_in_hospital_cardiac.16.aspx

Address for correspondence: Dr Elin Heledd Thomas, Morriston Hospital, Heol Maes Eglwys, Morriston, Swansea, SA6 6NL, UK.

Email: elinhthomas@doctors.org.uk 